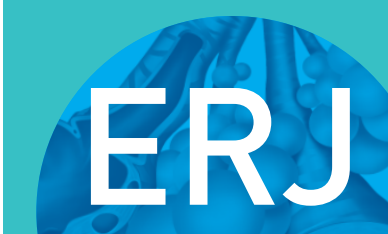

open research
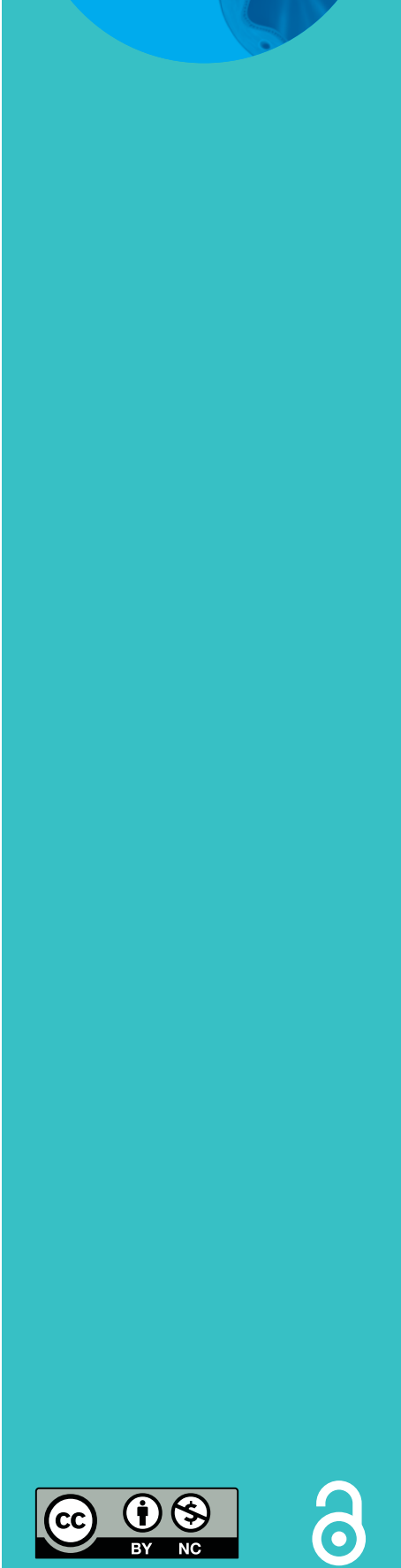

\section{A new mathematical model to identify contacts with recent and remote latent tuberculosis}

\author{
Gabrielle Fröberg (101,2, Emilie Wahren Borgström (10 ${ }^{1,2}$, Erja Chryssanthou ${ }^{3}$, \\ Margarida Correia-Neves ${ }^{1,4,5}$, Gunilla Källenius (i) ${ }^{1}$ and Judith Bruchfeld ${ }^{1,2}$
}

Affiliations: ${ }^{1}$ Division of Infectious Diseases, Dept of Medicine Solna, Karolinska Institutet, Stockholm, Sweden. ${ }^{2}$ Dept of Infectious Diseases, Karolinska University Hospital, Stockholm, Sweden. ${ }^{3}$ Dept of Clinical Microbiology, Karolinska University Hospital, Stockholm, Sweden. ${ }^{4}$ Life and Health Sciences Research Institute (ICVS), School of Medicine, University of Minho, Braga, Portugal. ${ }^{5}$ ICVS/3B'S, PT Government Associate Laboratory, Braga/Guimarães, Portugal.

Correspondence: Gabrielle Fröberg, B3:00 Dept of Infectious Diseases, Karolinska University Hospital, 17176 Stockholm, Sweden. E-mail: gabrielle.frobergasll.se

ABSTRACT Tuberculosis (TB) elimination programmes need to target preventive treatment to groups with an increased risk of $\mathrm{TB}$ activation, such as individuals with a latent tuberculosis infection (LTBI) acquired recently. Current diagnostic tests for LTBI have poor predictive values for TB activation and there is, at present, no reference method to evaluate new LTBI diagnostic and prognostic tools. Thus, our objective was to develop a mathematical model, independent of currently available diagnostic tests, to estimate the individual probability of recent and/or remote LTBI.

Estimations of recent LTBI were based on the contagiousness of index case, proximity and time of exposure, and environmental factors. Estimation of remote LTBI was based on country of origin, previous stays in high-risk environments or known exposure to TB. Individual probabilities were calculated and compared with tuberculin skin test (TST) and interferon- $\gamma$ release assay results for 162 contacts of 42 index TB cases.

Probabilities of remote LTBI were $16 \%$ for European/American contacts and 38\% for African/Asian contacts. The probability of recent LTBI was $35 \%$ for close contacts to smear microscopy positive index cases. A higher probability of remote LTBI was seen among TST-positive contacts.

This model may, with further validation, be used as an independent tool to evaluate new diagnostic markers for recent LTBI.

@ERSpublications

This mathematical model to estimate probability of recent and remote latent TB was based on clinical and epidemiological risk factors of exposure and may be used in the evaluation of new diagnostic markers with enhanced predictive values for TB activation http://bit.ly/2IWi6Ru

Cite this article as: Fröberg G, Wahren Borgström E, Chryssanthou E, et al. A new mathematical model to identify contacts with recent and remote latent tuberculosis. ERJ Open Res 2019; 5: 000782019 [https://doi.org/10.1183/23120541.00078-2019].

This article has supplementary material available from openres.ersjournals.com

Received: March 262019 | Accepted: April 102019

Copyright $\odot$ ERS 2019. This article is open access and distributed under the terms of the Creative Commons Attribution Non-Commercial Licence 4.0. 


\section{Introduction}

Today, tuberculosis (TB) is the number one infectious cause of mortality worldwide [1]. The World Health Organization has set the goal of substantially reducing the worldwide TB incidence by $90 \%$ by the year 2035 [2]. Up to a quarter of the world's population is estimated to be infected with latent TB (latent tuberculosis infection (LTBI)). $\sim 10 \%$ of these are estimated to progress to active TB $[3,4]$, mainly during the first 2 years after infection. As such, contacts of active TB cases and newly arrived migrants from highly TB-endemic countries are the main risk groups with an increased probability of activating LTBI in Europe. Other risk groups are individuals with an immunosuppressive condition (e.g. HIV or pregnancy) $[5,6]$ or treatment with tumour necrosis factor inhibitors, where there is also an increased probability of activating LTBI acquired more remotely [4]. TB elimination programmes specifically need to target groups with LTBI and an increased risk of TB activation to reduce numbers needed to treat (NNT) with preventive treatment.

Current diagnostic tools for LTBI, i.e. the tuberculin skin test (TST) and interferon- $\gamma$ release assays (IGRAs) such as QuantiFERON TB Gold In-Tube (QFT) (Qiagen, Hilden, the Netherlands) and T-spot.TB (Oxford Immunotec, Abingdon, UK), all detect immunological responses to Mycobacterium tuberculosis antigens but are neither able to distinguish between active TB and LTBI nor differentiate between a recently or a more remotely acquired LTBI. This is of special concern in contact screening of migrants from high TB burden countries with a high probability of being $M$. tuberculosis infected earlier in life. In addition, a problem in the development and evaluation of tests for LTBI is the lack of a reference method. To overcome this issue, prospective studies of TB activation in individuals with a positive or negative immune reactive test result have been performed, which require large cohorts and prolonged follow-up periods, and thus are both time consuming and costly. A large meta-analysis of TST and QFT predictive capacity have shown low positive predictive values (PPVs) for TB activation within 2 years (1.5\% and 2.7\%, respectively, overall and $2.4 \%$ and $6.8 \%$, respectively, in high-risk groups) [7]. Even for high QFT results $\left(>4 \mathrm{IU} \cdot \mathrm{mL}^{-1}\right)$, PPVs have been poor $(2.5 \%$ overall and $2.9 \%$ in patients with any medical risk factor) [8]. Low PPVs require high NNTs to prevent one case of active TB, especially when considering the total effectiveness of treatment, which also depends on efficacy and adherence. However, the negative predictive values (NPVs) of current standard tests for LTBI are very high $(>99 \%)$ in immunocompetent individuals. However, indeterminate and false negative results are more common in immunosuppressive conditions $[9,10]$ with an inherent risk of missing patients in need of preventive treatment.

Thus, there is an urgent need for tests with an improved capability to identify individuals with a recent LTBI and, as such, an increased probability of progression to active TB, i.e. a higher PPV.

Our objective was to develop a mathematical model, independent of currently available diagnostic tests, to estimate the individual probability of recent and/or remote LTBI, which may be used as a tool to evaluate new diagnostic markers with enhanced PPVs for TB activation.

\section{Material and methods}

The probability of becoming infected upon exposure to $M$. tuberculosis is mainly exogenous in nature, i.e. the summarised amount of $M$. tuberculosis inhaled, which depends on several features such as the contagiousness of the index case, proximity and time of exposure, and type of environment. The probability of developing active disease after infection is mainly endogenous in nature $[11,12]$, i.e. the host's immunological ability to control inhaled M. tuberculosis. The development of this model focused on the probability of being infected. TST or IGRA results were not included in the calculations, as the model is intended to be an independent tool to evaluate both existing and new immunological markers for LTBI.

\section{Definitions}

Remote LTBI was defined as an infection acquired previously, i.e. before the present contact with a defined index case with pulmonary TB.

Recent LTBI was defined as an infection acquired during the present contact with a defined index case with pulmonary TB.

\section{Calculations of the probability of remote LTBI}

Estimation of the probability of remote LTBI (premote) (expressed as a percentage) included the probability of infection in the country of origin (porigin), in Sweden (pSweden), during travel to highly endemic countries for $>1$ year (ptravel), during stays in high-risk environments such as hospitals, refugee camps and/or prisons for $>3$ months (phigh), and previous known exposure to contagious pulmonary TB (pexpo) (a more detailed report and an example of the calculations of premote is included in supplementary 
material S1):

$$
\text { premote }=1-((1-\text { porigin })(1-\text { psweden })(1-\text { ptravel })(1-\text { phigh })(1-\text { pexpo }))
$$

where:

$$
\text { porigin }=100\left(1-\left(1-\frac{\mathrm{ARI}}{100}\right)^{\text {years }}\right)
$$

for three separate time-periods (1990, 1990-2000 and 2000), and where "years" is number of years in the country of origin from birth to immigration to Sweden and ARI is the annual risk of infection:

$$
\mathrm{ARI}=\frac{\mathrm{TB} \text { incidence }}{\text { Dye's constant }}
$$

adjusted for country of origin and each time period [13-16].

pSweden, ptravel and phigh were calculated in similar ways with years in Sweden, during travel or staying in high-risk environments, and adjusted to Dye's constant, respectively.

pexpo was estimated according to previously published data [17], i.e. close contact with a smear microscopy positive $\left(\mathrm{SM}^{+}\right)$contact $35 \%$, smear microscopy negative $\left(\mathrm{SM}^{-}\right) 10 \%$ and smear microscopy result unknown $\left(\mathrm{SM}^{\text {unknown }}\right) 20 \%$, while not close (casual) contact with $\mathrm{SM}^{+} 10 \%, \mathrm{SM}^{-} 2 \%$ and $\mathrm{SM}^{\text {unknown }} 5 \%$.

\section{Calculations of probability of recent LTBI}

Estimations of the probability of recent LTBI (precent) (expressed as a percentage) included the contagiousness of index case, proximity and time of exposure to the index case, and environmental factors (figure 1) (a more detailed report and an example of the calculations of precent is included in supplementary material S2.

$$
\text { precent }=1-((1-\text { precent,day })(1-\text { precent,night }))
$$

precent,day and precent,night were calculated as $1-(1-\mathrm{pi})^{\mathrm{n}}$ according to daytime and night time parameters, respectively, where $\mathrm{n}$ is the estimated number of breaths taken by an individual contact during the estimated time of TB exposure and $\mathrm{pi}$ is the probability of the individual contact inhaling $M$. tuberculosis in the air volume with each breath:

$$
\mathrm{p}_{\mathrm{i}}=\frac{\mathrm{Dn}^{+} S V_{\mathrm{iK}}}{E V \mathrm{~d}}
$$

where $\mathrm{Dn}^{+}$is the estimated production of $M$. tuberculosis-containing droplets (Dn) per minute, calculated as:

$$
\mathrm{Dn}^{+}=C \mathrm{MtbDn}
$$

where CMtb is the M. tuberculosis concentration in sputum calculated as average number of colony forming units per microlitre of sputum [18, 19]. Dn is the estimated production of droplet nuclei adjusted for daytime/nighttime and with/without cough [20-25]. $S$ is the saturation of $\mathrm{Dn}^{+}$in air volume calculated from estimated elimination rate $(E)$ through ventilation and precipitation in general buildings or hospitals [11]. At steady state, $E=1 . V_{\mathrm{i}}$ is the estimated alveolar volume (in litres) in one normal inhalation by the contact $[26,27] . V \mathrm{~d}$ is the volume of distribution calculated as the estimated volume of air (in litres) of the premises in which the main exposure took place. $\mathrm{K}$ is a calculated constant that was applied to adjust for unknown parameters such as numbers of $M$. tuberculosis-containing droplets, $M$. tuberculosis per droplet, $M$. tuberculosis reaching the alveoli and M. tuberculosis needed to establish infection, and validated according to previous published data on transmission [17, 28, 29].

\section{Application of the mathematical model to contacts of contagious TB}

The mathematical model was applied to 162 contacts of 42 index cases with culture-verified pulmonary TB who were included in a study on new immunological markers for LTBI (Wahren Borgström et al. 2019; unpublished observations). In regular clinical practice, two contacts were diagnosed with active TB and therefore excluded from further analyses. 


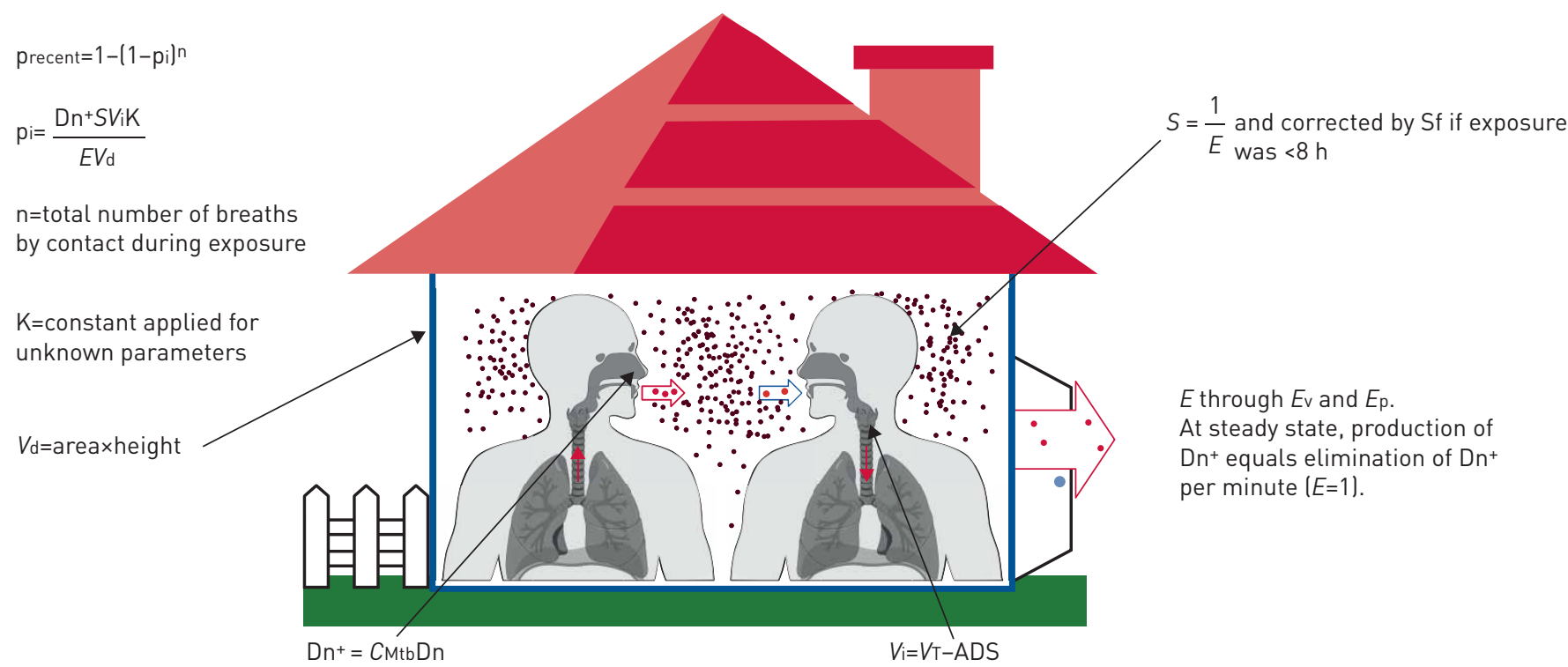

FIGURE 1 Illustration of the parameters included in the calculations of probability of recent latent tuberculosis infection (precent). pi: probability of the contact inhaling Mycobacterium tuberculosis in one breath; $V_{\mathrm{d}}$ : volume of distribution; $\mathrm{Dn}^{+}$: production of $M$. tuberculosis-containing droplets per minute; $C_{M t b}$ : concentration of M. tuberculosis in sputum; Dn: estimated droplet production; $V_{i}$ : volume of one inhalation; $V_{T}$ : total inhaled volume; ADS: anatomical dead space; $E$ : elimination rate of $\mathrm{Dn}^{+}$per minute; Ev: elimination through ventilation; Ep: elimination through precipitation; S: saturation at steady state, i.e. how many times more $\mathrm{Dn}^{+}$was present in the air as compared with the production per minute; Sf: saturation factor.

Index cases

31 (74\%) index cases were coughing, $24(57 \%)$ were $\mathrm{SM}^{+}$and average time from start of symptoms to treatment initiation was 81 days (range $27-313$ days).

\section{Contacts}

130 (81\%) contacts were bacille Calmette-Guérin vaccinated at least once.

With respect to origin, 70 (44\%) were Swedish, 10 (6\%) European excluding Sweden or American and 80 (50\%) were African or Asian. 26 (16\%) had previous known exposure to active TB and $26(16 \%)$ had previously been treated partly or fully for LTBI.

Proximity between index case and contact

$134(84 \%)$ were classified as close contacts $\left(>8 \mathrm{~h}\right.$ contact with $\mathrm{SM}^{+}$or $>48 \mathrm{~h}$ contact with $\mathrm{SM}^{-}$index case) and $26(16 \%)$ as casual contacts [17].

\section{Classification of probability groups}

Contacts were classified into four LTBI probability groups according to precent and premote: 1) recent LTBI, i.e. precent $\geqslant 10 \%$ and premote $<10 \%$; 2) remote LTBI, i.e. precent $<10 \%$ and premote $\geqslant 10 \%$; 3 ) recent and remote LTBI, i.e. precent $\geqslant 10 \%$ and premote $\geqslant 10 \%$; and 4 ) low probability of LTBI, i.e. precent $<10 \%$ and premote $<10 \%$. The same classification was performed with a probability cut-off at $50 \%$.

\section{Immunological test results}

Results of immune reactive testing with TST, QFT and T-spot.TB of all contacts were available, and the tests had been performed by nurses specifically trained in the technique. TST was performed $<1$ month after the last exposure date and repeated after 1-4 months if the initial test was negative, according to clinical standards. Two units of purified protein derivative (tuberculin PPD RT23; Statens Serum Institute, Copenhagen, Denmark) were injected intradermally and cutaneous induration was measured in millimetres with a ruler after $72 \mathrm{~h}$. The cut-off for a positive result was $10 \mathrm{~mm}$ with a borderline result of 6-9 mm [3, 30]. QFT and T-spot.TB were performed and analysed according to the manufacturers' instructions. For QFT, the defined cut-off for a positive test was $>0.7 \mathrm{IU} \cdot \mathrm{mL}^{-1}$ and a borderline result was defined as $0.2-0.7 \mathrm{IU} \cdot \mathrm{mL}^{-1}$ according to a recent study on serial QFT testing [31]. For T-spot.TB, the responses to the $M$. tuberculosis antigens $6-\mathrm{kDa}$ early secretory antigenic target (ESAT-6) and $10-\mathrm{kDa}$ culture filtrate protein (CFP-10) were analysed and presented separately to detect a possible difference in 
the response in recent versus remote LTBI. The defined cut-off for a positive test was $>7$ spots and a borderline result was defined as 5-7 spots according to the manufacturer's instructions.

\section{Classification of response groups}

Contacts were classified into five response groups according to immunological test results during follow-up: 1) negative response (TST $<6 \mathrm{~mm}$, QFT $<0.2 \mathrm{IU} \cdot \mathrm{mL}^{-1}$, or T-spot.TB ESAT-6 and CFP-10 $<5$ spots) $\geqslant 2$ months after exposure and over the 12-month follow-up if available; 2) borderline response (maximum TST 6-9 mm, QFT 0.2-0.7 IU.mL ${ }^{-1}$, or T-spot.TB ESAT-6 and CFP-10 5-7 spots) over the 12-month follow-up; 3) positive response (TST $>9 \mathrm{~mm}$, QFT $>0.7 \mathrm{IU} \cdot \mathrm{mL}^{-1}$, or T-spot.TB ESAT-6 and CFP-10 $>7$ spots) from $\leqslant 1$ month and over the 12-month follow-up if available; 4 ) conversion (TST/IGRA negative to positive or borderline to positive with TST $\geqslant 10 \mathrm{~mm}$ increase, QFT $\geqslant 0.5 \mathrm{IU} \cdot \mathrm{mL}^{-1}$ increase or T-spot.TB $\geqslant 3$ spot increase over a follow-up period of 12 months); and 5) reversion (TST/IGRA positive to negative or positive to borderline with TST $\geqslant 10 \mathrm{~mm}$ decrease, QFT $\geqslant 0.5 \mathrm{IU} \cdot \mathrm{mL}^{-1}$ decrease or T-spot.TB $\geqslant 3$ spot decrease over a follow-up period of 12 months). Undefined responses were excluded from analyses (e.g. borderline/positive results not classified as conversions/reversions, and unapproved or missing results).

\section{Statistical analyses}

Calculations were based on basic epidemiological concepts [32]. Distributions of precent and premote were nonparametric, and average probabilities are presented as median values. Comparison of precent and premote between defined groups were calculated by one-way ANOVA on ranks (Kruskal-Wallis) and Dunn's pair-wise test. Statistical significance was defined as $\mathrm{p} \leqslant 0.05$.

\section{Ethical clearance}

The study on new immunological markers for LTBI was approved by the ethical Committee in Stockholm (Dnr 2008/1208-31/3). Written and oral consent was obtained from all participants.

\section{Results}

premote in relation to contacts' country of origin

premote was $11 \%$ (interquartile range (IQR) 1-38\%) among all contacts (figure 2), 1\% (IQR 0.5-1\%) among contacts originating from Sweden $(\mathrm{p}<0.001), 16 \%$ (IQR 3-26\%) among contacts from Europe (excluding Sweden) and America, and 38\% (IQR 22-49\%) among contacts from Africa and Asia $(\mathrm{p}<0.001)$.

precent in contacts in relation to proximity to and sputum smear result of index cases.

precent among close contacts of $\mathrm{SM}^{+}$index cases was 35\% (IQR 6-74\%) (figure 3), as compared with $1 \%$ (IQR $0.3-3 \%$ ) among close contacts to $\mathrm{SM}^{-}$index cases $(\mathrm{p}<0.001), 2 \%$ (IQR $0.2-6 \%$ ) among casual

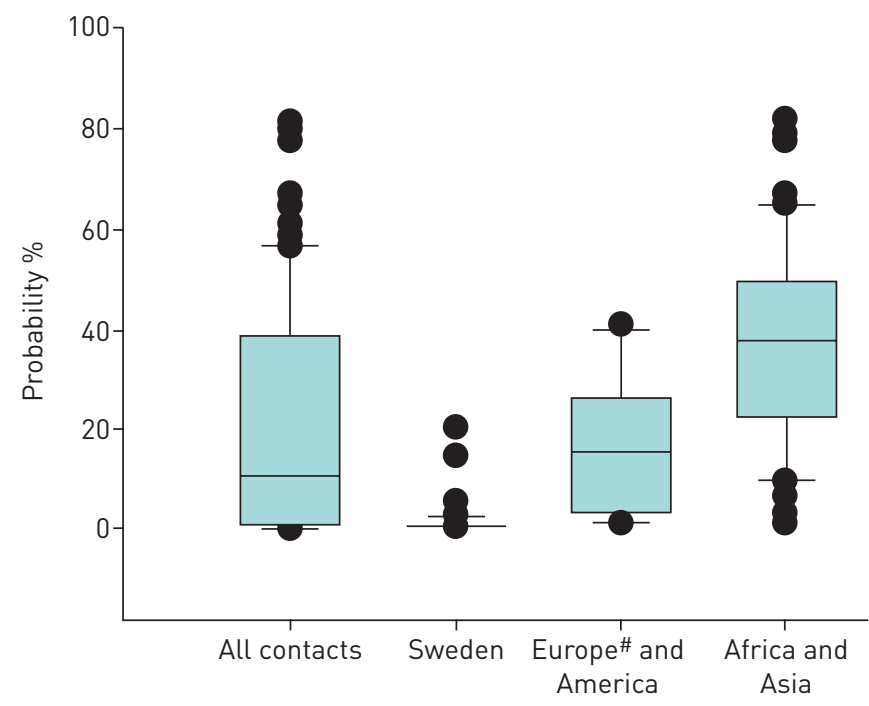

FIGURE 2 Probability of remote latent tuberculosis infection in contacts of pulmonary tuberculosis cases according to their regional origin. Horizontal lines represent median values, boxes represent interquartile ranges, whiskers represent the 10th and 90th percentiles, and circles represent outliers. \#: excluding contacts originating from Sweden. 


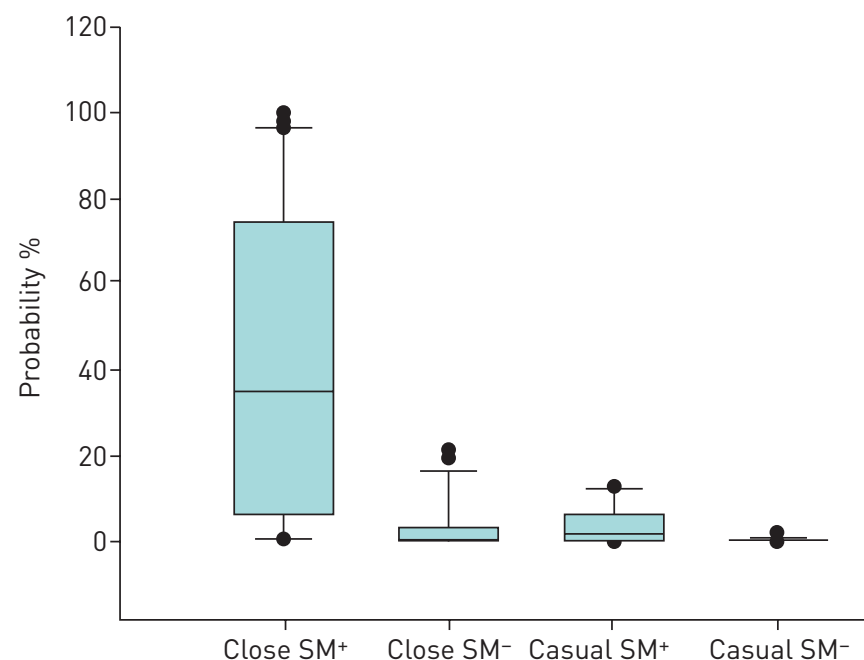

FIGURE 3 Probability of recent latent tuberculosis infection in contacts of pulmonary tuberculosis cases according to proximity to and sputum smear result of the index cases. Horizontal lines represent median values, boxes represent interquartile ranges, whiskers represent the 10th and 90th percentiles, and circles represent outliers. Close contacts: $>8 \mathrm{~h}$ contact with smear microscopy-positive $\left(\mathrm{SM}^{+}\right)$or $>48 \mathrm{~h}$ contact with smear microscopy-negative $\left(\mathrm{SM}^{-}\right)$index case; casual contacts: $<8 \mathrm{~h}$ contact with $\mathrm{SM}^{+}$or $<48 \mathrm{~h}$ contact with $\mathrm{SM}^{-}$index case [17].

contacts to $\mathrm{SM}^{+}$index cases $(\mathrm{p}=0.003)$ and $0.1 \%$ (IQR $\left.0.04-0.6 \%\right)$ among casual contacts to $\mathrm{SM}^{-}$index cases $(\mathrm{p}<0.001)$.

\section{Proportion of contacts classified into defined probability groups}

With a cut-off at $\geqslant 10 \%$ for high probability, $68 \%$ were classified as recent and/or remote LTBI (figure 4 ). With a cut-off at $\geqslant 50 \%$, only $38 \%$ were classified as recent and/or remote LTBI.

\section{Proportion and concordance of contacts classified into defined TST and IGRA response groups} The proportion of contacts in the TST and IGRA response groups (figure 5) and their mutual concordance was analysed to evaluate their variability in detecting an immunological response, which has to be considered when evaluating calculated probabilities in relation to these tests. Contacts in the defined response groups borderline, reversions or undefined were not included.

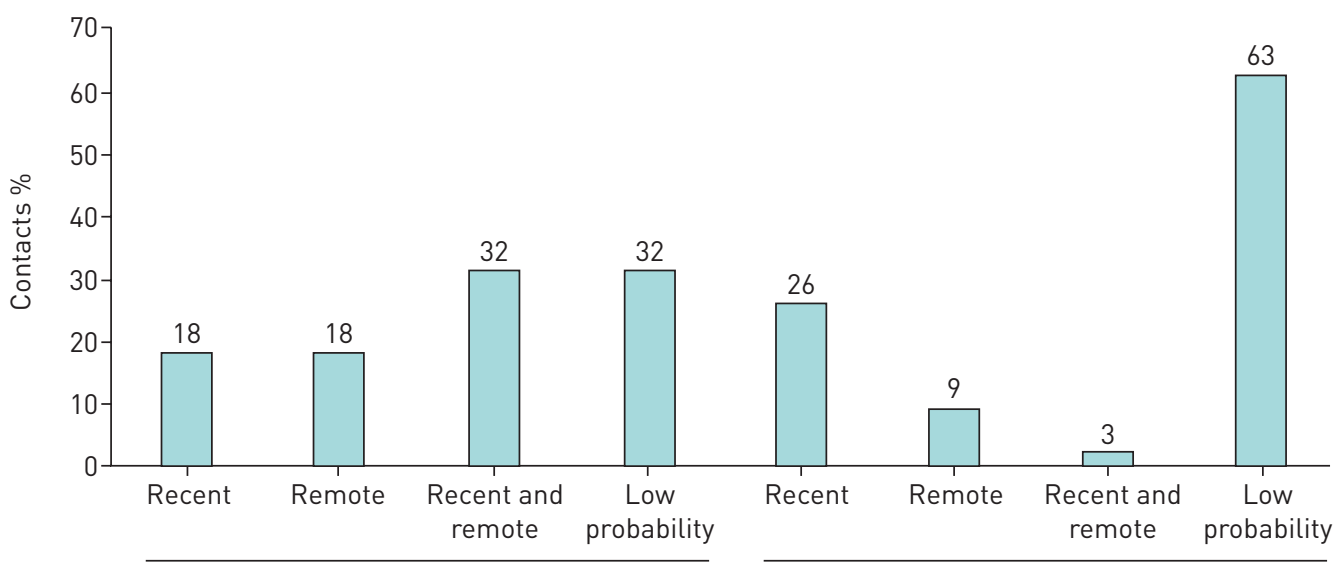

Cut-off $10 \%$

Cut-off $50 \%$

FIGURE 4 Proportion of contacts of pulmonary tuberculosis cases $(n=160)$ classified into defined probability groups with a cut-off for high probability at $\geqslant 10 \%$ or $\geqslant 50 \%$. Recent: $\geqslant 10 / 50 \%$ probability of recent and $<10 / 50 \%$ probability of remote latent tuberculosis infection (LTBI); remote; $<10 / 50 \%$ probability of recent and $\geqslant 10 / 50 \%$ probability of remote $\mathrm{LTBI}$; recent and remote: $\geqslant 10 / 50 \%$ probability of recent and $\geqslant 10 / 50 \%$ probability of remote $\mathrm{LTBI}$; low probability: $<10 / 50 \%$ probability of recent and $<10 / 50 \%$ probability of remote LTBI. 


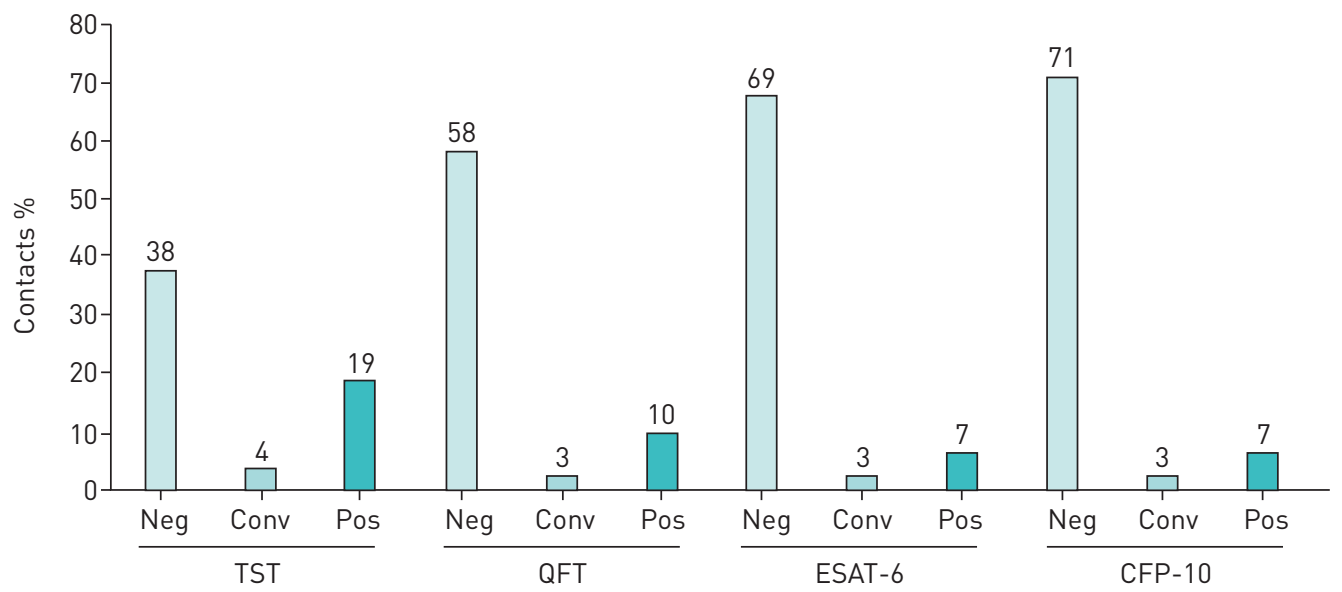

FIGURE 5 Proportion of contacts of pulmonary tuberculosis cases $(n=160)$ classified by tuberculin skin test (TST), QuantiFERON TB Gold In-Tube (QFT), Tspot.TB/6-kDa early secretory antigenic target (ESAT-6) and T-spot. TB/10-kDa culture filtrate protein (CFP-10) response groups defined as follows. Neg: negative test result at $\geqslant 2$ month; conv: conversion from a negative to a positive result over 12 months; pos: positive test result from $\leqslant 1$ month and no following reversion. Borderline results, reversions and undefined results are not presented herein.
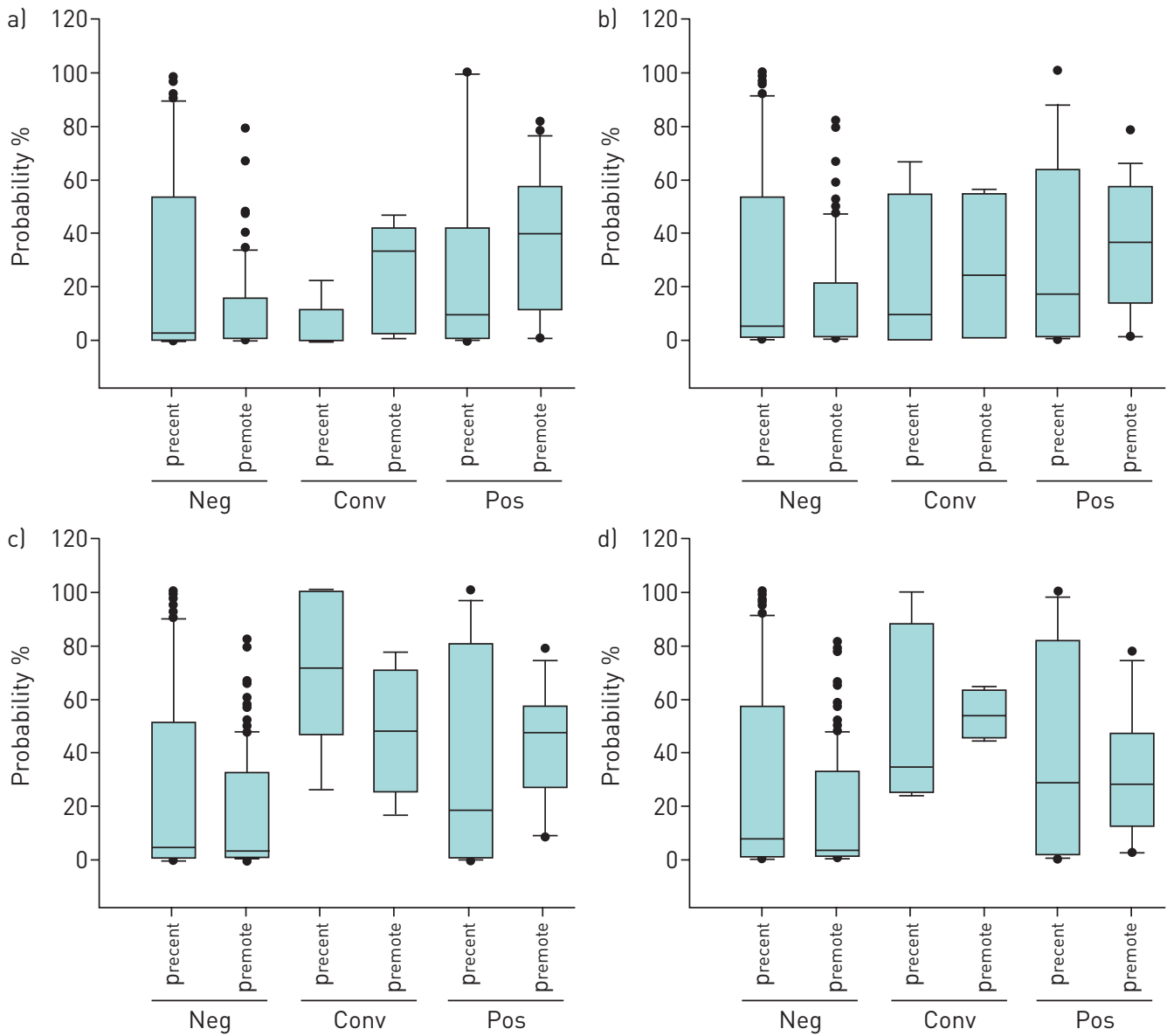

FIGURE 6 Probability of recent (precent) and remote (premote) latent tuberculosis infection in a) tuberculin skin test, b) QuantiFERON TB Gold In-Tube, c) T-spot. TB/6-kDa early secretory antigenic target and d) T-spot. TB/ $10-\mathrm{kDa}$ culture filtrate protein response groups defined as follows. Neg: negative test result at $\geqslant 2$ month; conv: conversion from a negative to a positive result over 12 months; pos: positive test result from $\leqslant 1$ month and no following reversion. Horizontal lines represent median values, boxes represent interquartile ranges, whiskers represent the 10th and 90th percentiles, and circles represent outliers. 
Concordant responses were highest between QFT and ESAT-6 (95\%), QFT and CFP-10 (94\%), and ESAT-6 and CFP-10 (94\%), while concordant response was lower between TST and QFT (79\%), TST and ESAT-6 (77\%), as well as TST and CFP-10 (73\%).

precent and premote in relation to TST and IGRA response groups.

There was no statistically significant difference between the precent as compared to premote in either response groups (figure 6).

premote was higher in contacts with a TST positive than negative response ( $40 \%$ versus $1 \%, \mathrm{p}<0.001)$. There was a tendency for higher precent in contacts with T-spot.TB/ESAT-6 conversion than negative response ( $72 \%$ versus $5 \%)$; however, the numbers were small and did not reach statistical significance $(\mathrm{p}=0.07)$.

We calculated on the sensitivity, specificity, PPV and NPV of high probability (cut-offs $\geqslant 10 \%$ or $\geqslant 50 \%$ ) of recent and/or remote LTBI to detect a positive response, or a conversion in TST or IGRA. Results for TST and IGRAs are presented separately and in combination, i.e. positive result and/or conversion in any test, as well as negative results in all tests (table 1).

With a $10 \%$ cut-off, sensitivity of high probability to detect a positive result or conversion in ESAT- 6 and CFP-10 was $100 \%$, although specificity was generally $<50 \%$. The PPV of high probability was highest for prediction of a positive result or conversion of TST, i.e. $47 \%$. With a cut-off at $50 \%$, sensitivity was generally $<70 \%$, while PPV was as high as $58 \%$ for TST.

\section{Discussion}

With the herein presented mathematical model, premote was in line with published epidemiological data (www.who.int/tb/country/data/download/en/) [13,33]. precent among close contacts of $\mathrm{SM}^{+}$index cases was also in line with published data $[12,17,18,34-36]$. However, even though our estimations were thorough and in line with previous data, other factors may also influence and change over time (e.g. urban or rural settings and socioeconomic standards). Furthermore, several approximations had to be made (e.g. production of droplets in correlation to activity and cough, M. tuberculosis-containing droplets, number of $M$. tuberculosis needed to establish infection, time and place spent together with index, and extent of ventilation). Anatomical airway defences and the innate immune response and microbiota may also influence the response to M. tuberculosis $[29,37]$. Some of the features can be updated and refined with further validation of the model, such as extent and type of cough, and ventilation in different buildings (i.e. apartment/house/public space/hospital), and maybe also adjusted for additional ventilation by open windows. However, a mathematical model will never be absolute and must also be applicable in clinical practice.

Previous models have been described for the prediction of LTBI. BAILEY et al. [38] were able to predict a positive TST in contacts of active TB cases with $89 \%$ sensitivity, $36 \%$ specificity and $26 \% \mathrm{PPV}$. The corresponding figures in our model were $80 \%, 48 \%$ and $47 \%$ for TST with $10 \%$ probability cut-off, and

TABLE 1 Sensitivity, specificity, positive predicted value (PPV) and negative predicted value (NPV) of high probability for recent and/or remote latent tuberculosis infection (cut-offs $\geqslant 10 \%$ or $\geqslant 50 \%$ ) to detect a positive response or a conversion in the tuberculin skin test (TST) and interferon- $y$ release assays

\begin{tabular}{lccccc} 
Cut-off & Test & Sensitivity & Specificity & PPV & NPV \\
\hline $\mathbf{1 0} \%$ & TST & $80 \%$ & $48 \%$ & $47 \%$ & $81 \%$ \\
& QFT & $85 \%$ & $40 \%$ & $23 \%$ & $93 \%$ \\
& ESAT-6 & $100 \%$ & $36 \%$ & $19 \%$ & $100 \%$ \\
& CFP-10 & $100 \%$ & $35 \%$ & $17 \%$ & $100 \%$ \\
$\mathbf{5 0} \%$ & All & $88 \%$ & $39 \%$ & $25 \%$ & $94 \%$ \\
& TST & $54 \%$ & $71 \%$ & $58 \%$ & $67 \%$ \\
& QFT & $50 \%$ & $69 \%$ & $26 \%$ & $87 \%$ \\
& ESAT-6 & $69 \%$ & $66 \%$ & $23 \%$ & $94 \%$ \\
& CFP-10 & $60 \%$ & $65 \%$ & $18 \%$ & $92 \%$ \\
& All & $57 \%$ & $67 \%$ & $31 \%$ & $86 \%$ \\
\hline
\end{tabular}

Results for TST and interferon-y release assays are presented separately and in combination, i.e. positive result and/or conversion in any test, as well as negative result in all tests. QFT: QuantiFERON TB Gold In-Tube; ESAT-6: 6-kDa early secretory antigenic target; CFP-10: 10-kDa culture filtrate protein. 
$54 \%, 71 \%$ and $58 \%$ for TST with $50 \%$ probability cut-off. This may be interpreted as TST performing poorly in the diagnosis of LTBI. However, with a cut-off at $10 \%$, the probabilities among contacts of being infected are $10-100 \%$ and the corresponding probabilities of not being infected are thus $0-90 \%$. With a cut-off at 50\%, the PPV increases but still, many in this group will not be infected. A direct comparison between probability of infection and positive test rates would be desirable but this requires a highly reliable test as a reference method. In the absence of such a method, we do not know the true performance of TST or IGRA in detecting LTBI. However, a negative TST/IGRA result in an immunocompetent individual is associated with high NPV, i.e. excludes LTBI with high accuracy. Thus, this model is constructed to be independent of TST/IGRA results with the aim to be a new tool to evaluate new tests with better capacity to predict true LTBI with remaining $M$. tuberculosis. Therefore, comparisons between calculated probabilities and TST/IGRA results in this study should not be interpreted as a validation of the model.

Another published model is the McGill web-based algorithm (www.tstin3d.com/en/calc.html), which provides a tool to calculate the probability that a positive TST and/or QFT represent a true LTBI, and the calculated life-long risk of TB activation as well as the risk of liver toxicity with 9 months of daily isoniazid [39]. However, results from this algorithm do not differentiate between a recent or a remote LTBI, which is often the dilemma in clinical practice as well as in research on diagnostic markers of LTBI.

Our mathematical model is a bottom-up construction based on theoretical arguments, estimations and previous studies on transmission, which may suggest that it may be hard to determine the validity. However, we consider the theoretical approach as a strength, as the model is totally transparent and adjustable, not only to updated and optimised parameters but also to empirical data from larger cohorts with extended follow-up, including a large number of TB contacts converting in TST/IGRA or activating TB.

Acknowledgements: A special thanks to Hans Gaines (The Swedish Institute for Infectious Disease Control, Solna, Sweden) for initiating and assisting in the mathematical concept of this model.

Conflict of interest: None declared.

Support statement: This work was supported by the Karolinska Institutet and Stockholm County (grants K0190-2014 and K2017-4578. Funding information for this article has been deposited with the Crossref Funder Registry.

\section{References}

World Health Organization. Global Tuberculosis Report. Geneva, WHO, 2017.

World Health Organization. End TB Strategy. Geneva, WHO, 2014.

World Health Organization. Guidelines on the Management of Latent Tuberculosis Infection. Geneva, WHO, 2015.

4 World Health Organization. Latent TB Infection: Updated and Consolidated Guidelines for Programmatic Management. Geneva, WHO, 2018.

5 Kidd P. Th1/Th2 balance: the hypothesis, its limitations, and implications for health and disease. Altern Med Rev 2003; 8: 223-246.

6 Yip L, McCluskey J, Sinclair R. Immunological aspects of pregnancy. Clin Dermatol 2006; 24: 84-87.

7 Diel R, Loddenkemper R, Nienhaus A. Predictive value of interferon-gamma release assays and tuberculin skin testing for progression from latent TB infection to disease state: a meta-analysis. Chest 2012; 142: 63-75.

8 Winje BA, White R, Syre H, et al. Stratification by interferon- $\gamma$ release assay level predicts risk of incident TB Thorax 2018; in press [https://doi.org10.1136/thoraxjnl-2017-211147].

9 Dominguez J, Latorre I, Altet N, et al. IFN- $\gamma$-release assays to diagnose TB infection in the immunocompromised individual. Expert Rev Respir Med 2009; 3: 309-327.

10 Sester M, van Leth F, Bruchfeld J, et al. Risk assessment of tuberculosis in immunocompromised patients. A TBNET study. Am J Respir Crit Care Med 2014; 190: 1168-1176.

11 Rieder HL. Epidemiologic basis of tuberculosis control. Nat Genet; 1999; 22: 59-62.

12 Sepkowitz KA. How contagious is tuberculosis? Clin Infect Dis 1996; 23: 954-962.

13 Dye C, Scheele S, Dolin P, et al. Global burden of tuberculosis: estimated incidence, prevalence, and mortality by country. JAMA 1999; 282: 677-686.

14 Dye C. Breaking a law: tuberculosis disobeys Styblo's rule. Bull World Health Organ 2008; 86: 4.

15 Dye C, Bassili A, Bierrenbach AL, et al. Measuring tuberculosis burden, trends, and the impact of control programmes. Lancet Infect Dis 2008; 8: 233-243.

16 Kompala T, Shenoi SV, Friedland G. Transmission of tuberculosis in resource-limited settings. Curr HIV/AIDS Rep 2013; 10: 264-272.

17 Grzybowski S, Barnett GD, Styblo K. Contacts of cases of active pulmonary tuberculosis. Bull Int Union Tuberc 1975; 50: 90-106.

18 Lohmann EM, Koster BF, le Cessie S, et al. Grading of a positive sputum smear and the risk of Mycobacterium tuberculosis transmission. Int J Tuberc Lung Dis 2012; 16: 1477-1484.

19 Andersen AA. New sampler for the collection, sizing, and enumeration of viable airborne particles. $J$ Bacteriol 1958; 76: 471-484.

20 Johnson GR, Morawska L. The mechanism of breath aerosol formation. J Aerosol Med Pulm Drug Deliv 2009; 22 229-237.

21 Nicas M, Nazaroff WW, Hubbard A. Toward understanding the risk of secondary airborne infection: emission of respirable pathogens. J Occup Environ Hyg 2005; 2: 143-154.

22 Douglas NJ, White DP, Pickett CK, et al. Respiration during sleep in normal man. Thorax 1982; 37: 840-844. 
23 Fennelly KP, Martyny JW, Fulton KE, et al. Cough-generated aerosols of $M$
method to study infectiousness. Am J Respir Crit Care Med 2004; 169: 604-609.
24 Fennelly KP, Jones-Lopez EC, Ayakaka I, et al. Variability of infectious aerosoli

Fennelly KP, Jones-Lopez EC, Ayakaka I, et al. Variability of infectious aerosols produced during coughing by patients with pulmonary tuberculosis. Am J Respir Crit Care Med 2012; 186: 450-457. Jones-Lopez EC, Namugga O, Mumbowa F, et al. Cough aerosols of Mycobacterium tuberculosis predict new infection: a household contact study. Am J Respir Crit Care Med 2013; 187: 1007-1015.

26 Quanjer PH, Stanojevic S, Cole TJ, et al. Multi-ethnic reference values for spirometry for the 3-95-yr age range: the global lung function 2012 equations. Eur Respir J 2012; 40: 1324-1343.

27 Hart MC, Orzalesi MM, Cook CD. Relation between anatomic respiratory dead space and body size and lung volume. J Appl Physiol 1963; 18: 519-522.

28 Fennelly KP, Jones-Lopez EC. Quantity and quality of inhaled dose predicts immunopathology in tuberculosis. Front Immunol 2015; 6: 313.

29 Gupta N, Kumar R, Agrawal B. New players in immunity to tuberculosis: the host microbiome, lung epithelium, and innate immune cells. Front Immunol 2018; 9: 709.

30 Erkens CG, Kamphorst M, Abubakar I, et al. Tuberculosis contact investigation in low prevalence countries: a European consensus. Eur Respir J 2010; 36: 925-949.

31 Nemes E, Rozot V, Geldenhuys H, et al. Optimization and interpretation of serial QuantiFERON testing to measure acquisition of Mycobacterium tuberculosis infection. Am J Respir Crit Care Med 2017; 196: 638-648.

32 Bonita R, Beaglehole R, Kjellström T. Basic Epidemiology. 2nd Edn. Geneva, WHO, 2006.

33 Glaziou P, Floyd K, Raviglione M. Global burden and epidemiology of tuberculosis. Clin Chest Med 2009; 30: 621-636

34 Langenskiold E, Herrmann FR, Luong BL, et al. Contact tracing for tuberculosis and treatment for latent infection in a low incidence country. Swiss Med Wkly 2008; 138: 78-84.

35 Marks SM, Taylor Z, Qualls NL, et al. Outcomes of contact investigations of infectious tuberculosis patients. Am J Respir Crit Care Med 2000; 162: 2033-2038.

36 Sprinson JE, Flood J, Fan CS, et al. Evaluation of tuberculosis contact investigations in California. Int J Tuberc Lung Dis 2003; 7: 12 Suppl. 3, S363-S368.

37 Simmons JD, Stein CM, Seshadri C, et al. Immunological mechanisms of human resistance to persistent Mycobacterium tuberculosis infection. Nat Rev Immunol 2018; 18: 575-589.

38 Bailey WC, Gerald LB, Kimerling ME, et al. Predictive model to identify positive tuberculosis skin test results during contact investigations. JAMA 2002; 287: 996-1002.

39 Menzies D, Gardiner G, Farhat M, et al. Thinking in three dimensions: a web-based algorithm to aid the interpretation of tuberculin skin test results. Int J Tuberc Lung Dis 2008; 12: 498-505. 